\title{
A NOTE ON ŠNIREL'MAN'S APPROACH TO GOLDBACH'S PROBLEM
}

\author{
R. C. VAUGHAN
}

\section{Introduction and statement of theorem}

The object of this note is to demonstrate that Šnirel'man's method [38], [39] (see also Landau [22], [23]) as modified by Shapiro and Warga [36] will give the following theorem.

THEOREM. Every sufficiently large odd number can be written as the sum of five odd prime numbers, and thus every sufficiently large number is the sum of at most six prime numbers.

It is apparent from the proof that the method only just misses giving four for even numbers, and that is the best that has been obtained for even numbers by the much more powerful Hardy-Littlewood-Vinogradov method (see I. M. Vinogradov [42], [43], [44]).

In [38] Šnirel'man has a large positive constant in place of the six, and this has been reduced successively to 2208 by Romanov [35], to 71 by Heilbronn, Landau and Scherk [17], 67 by Ricci [32], [33], 20 by Shapiro and Warga [36], 18 by Yin [45], 12 by Klimov and Kondakova [20] and 10 by Čečuro and Kuzjašev [5] and Siebert [37] independently. Čečuro and Kuzjašev, and Siebert use the Bombieri-A. I. Vinogradov theorem [4], [41], and in view of the work of Linnik [25], [26], Čudakov [8] and Montgomery [29, Chapter 16] we know that this is of about the same "depth" as the Hardy-Littlewood-Vinogradov method.

The argument presented here does not use the Bombieri-Vinogradov theorem, but depends instead on a theorem of Davenport and Halberstam [10] (see also Barban [1], [2], [3, Theorem 3.2] and Gallagher [14, Theorem 3]) which is a simple consequence of the Siegel-Val'fis theorem and the large sieve. In principle, the new idea is that counting all the numbers in an interval which are representable as the sum of two prime numbers enables one to average over all the residue classes of all the sifting moduli. One of the fascinating aspects of this subject is the interrelation between the various methods. Montgomery's later sharpening [28], [29, Chapter 17] of the Davenport-Halberstam theorem involved the Hardy-Littlewood-Vinogradov method, but Hooley has recently shown [18] that estimates for exponential sums involving primes, of the Vinogradov type, can be avoided and indeed obtains still sharper results.

Although largely superseded by I. M. Vinogradov's work, the Šnirel'man method has the advantage that it is relatively easy to compute an $s$ such that every even number is the sum of at most $s$ prime numbers. Klimov [19] has given $s=6 \times 10^{9}$ and more recently, with Pil'tjail and Septickaja [20a] has reduced this to $s=115 . \dagger$

\section{Received 27 June 1975}

$\dagger$ Note added in proof: This can be reduced further to 27. The proof is to appear in J. Reine Angew. Math.

[Bull. London Math. Soc., 8 (1976), 245-250] 
Lavrik has observed [24] that the original Šnirel'man device of using Cauchy's inequality

$$
\left(\sum_{n \leqslant x} r(n)\right)^{2} \leqslant \sum_{\substack{m \leqslant x \\ r(m)>0}} \sum_{n \leqslant x} r(n)^{2}
$$

where $r(n)$ is the number of representations of $n$ as the sum of two prime numbers, will never enable one to do better than the above theorem with six replaced by eight or so. Instead we follow in the spirit of Shapiro and Warga [36], who applied the Selberg sieve directly to a sum of the kind $\sum_{n \leqslant x} r(n) a_{n}$, where the $a_{n}$ are suitable weights which smooth out the irregularities of $r(n)$. For some brief comments on the method see Čudakov and Klimov [9].

\section{Proof of the theorem}

Let $x$ be a large real number,

$$
\begin{aligned}
& R(n)=\sum_{3 \leqslant p_{1} \leqslant x} \sum_{\substack{p_{2} \geqslant 3 \\
p_{1}+p_{2}=n}} \log p_{1}, \\
& f_{n}(p)=\left\{\begin{array}{cc}
0 & p \mid n \\
\frac{1}{p-2} & p \nmid n
\end{array}\right\}(2 \mid n), \quad f_{n}(q)=\mu(q)^{2} \prod_{p \mid q} f_{n}(p), \\
& \vartheta(x)=\sum_{3 \leqslant p \leqslant x} \log p, \vartheta(x, d, n)=\sum_{\substack{3 \leqslant p \leqslant x \\
p \equiv n(\bmod d)}} \log p,
\end{aligned}
$$

and

$$
\mathscr{L}=\log x, \quad y=x \mathscr{L}^{-20}, \quad \xi=y^{\frac{1}{2}},
$$

where $\mu$ is the Möbius function. Then it is a straightforward consequence of Selberg's upper bound sieve method (see, for example, Theorem 3.2 of [15]. Note that the weights $\log p$ in (3) make no essential difference to the argument.) that

$$
R(n) \leqslant \frac{\vartheta(x)}{\sum_{q \leqslant \xi} f_{n}(q)}+y^{\frac{1}{2}} \log x+E(n) \quad(n>x, 2 \mid n)
$$

where

$$
E(n)=\sum_{\substack{d \leq y=1 \\(d, n)=1}} 3^{\omega(d)}\left|\vartheta(x, d, n)-\frac{\vartheta(x)}{\phi(d)}\right|,
$$

$\omega(d)$ denotes the number of different prime divisors of $d$, and $\phi$ is Euler's function.

By (6),

$$
\sum_{\substack{x<n \leqslant 2 x \\ 2 \mid n}} E(n) \ll x \sum_{d \leqslant y} 3^{\omega(d)} d^{-1} \sum_{\substack{n=1 \\(n, d)=1}}^{d}\left|\vartheta(x, d, n)-\frac{\vartheta(x)}{\phi(d)}\right|
$$


so that by Cauchy's inequality

$$
\left(\sum_{\substack{x<n \leqslant 2 x \\ 2 \mid n}} E(n)\right)^{2} \ll x^{2}\left(\prod_{p \leqslant y}\left(1+\frac{9}{p-1}\right)\right) \sum_{\substack{d \leqslant y \\ d \leqslant y=1}} \sum_{\substack{n=1 \\(n, d)=1}}^{d}\left|\vartheta(x, d, n)-\frac{\vartheta(x)}{\phi(d)}\right|^{2}
$$

By elementary prime number theory

$$
\prod_{p \leqslant y}\left(1+\frac{9}{p-1}\right) \ll \mathscr{L}^{9}
$$

and

$$
\sum_{d \leqslant y} \sum_{\substack{n=1 \\(n, d)=1}}^{d}\left|\vartheta(x, d, n)-\psi(x, d, n)-\frac{\vartheta(x)-\psi(x)}{\phi(d)}\right|^{2} \ll y x^{\frac{1}{2}} \mathscr{L}
$$

where as usual

$$
\psi(x)=\sum_{m \leqslant x} \Lambda(m), \psi(x, d, n)=\sum_{\substack{m \leqslant x \\ m \equiv n(\bmod d)}} \Lambda(m)
$$

with $\Lambda$ von Mangoldt's function. Hence, by (7) and Theorem 3 of Gallagher [14] (by slightly altering the value of $y$ one could equally well use the DavenportHalberstam theorem [10]),

$$
\sum_{\substack{x<n \leqslant 2 x \\ 2 \mid n}} E(n) \ll x^{2} \mathscr{L}^{-5}
$$

By (2) and (4),

$$
\sum_{q \leqslant \xi} f_{n}(q) \ll \mathscr{L}^{2}
$$

and, by (1), $R(n)=0$ if $2 \times n$. Hence, by (4), (5) and (8),

$$
\sum_{x<n \leqslant 2 x} R(n) \sum_{q \leqslant \xi} f_{n}(q) \leqslant \sum_{\substack{x<n \leqslant 2 x \\ R(n)>0}} \vartheta(x)+O\left(x^{2} \mathscr{L}^{-3}\right) .
$$

The next step is to estimate the left of (9) from below. By (2),

$$
\sum_{q \leqslant \xi} f_{n}(q) \geqslant\left(\prod_{\substack{p \mid n \\ p>2}} \frac{p-2}{p-1}\right) \sum_{\substack{q \leqslant \xi \\ q \text { odd }}} \frac{\mu(q)^{2}}{\phi_{1}(q)}
$$

where

$$
\phi_{1}(q)=\prod_{p \mid q}(p-2)
$$


Thus, by (1),

$$
\sum_{x<n \leqslant 2 x} R(n) \sum_{q \leqslant \xi} f_{n}(q) \geqslant\left(\sum_{\substack{q \leqslant \xi \\ q \text { odd }}} \frac{\mu(q)^{2}}{\phi_{1}(q)}\right) \sum_{\substack{r \leqslant 2 x \\ r \text { odd }}} \frac{\mu(r)}{\phi(r)} \sum_{\substack{p_{1} \\ 3 \leqslant p_{1} \leqslant x}} \sum_{\substack{p_{1} \geqslant 3 \\ p_{1} \geqslant p_{1}+p_{2} \\ x-p_{1}<p_{2} \leqslant 2 x-p_{1}}} \log p_{1} .
$$

When $r>\mathscr{L}^{10}$ one uses the crude estimate

$$
\sum_{\substack{p_{2} \geqslant 3 \\ r p_{1}+p_{2} \\ x-p_{1}<p_{2} \leqslant 2 x-p_{1}}} 1 \leqslant 1+\frac{x}{r}
$$

and when $r \leqslant \mathscr{L}^{10}$ one observes that the number of different prime divisors of $r$ is $\ll \mathscr{L}$. Hence, by (4) and the Siegel-Val'fiš theorem (c.f. $\$ 22$ of [11]),

$$
\begin{aligned}
\sum_{\substack{r \leqslant 2 x \\
r \text { odd }}} \frac{\mu(r)}{\phi(r)} \sum_{\substack{p_{1} \\
3 \leqslant p_{1} \leqslant x}} \sum_{\substack{p_{2} \geq 3 \\
\text { ond } \\
p_{1}+p_{2} \\
x-p_{1}<p_{2} \leqslant 2 x-p_{1}}} \log p_{1} \\
=\left(\prod_{p^{\prime} 2}\left(1-\frac{1}{(p-1)^{2}}\right)\right) \int_{3}^{2 x} \frac{\min (u, 2 x-u)}{\log u} d u+O\left(x^{2} \mathscr{L}^{-4}\right) .
\end{aligned}
$$

By (4) and (11),

$$
\sum_{\substack{q \leq \xi \\ q \text { odd }}} \frac{\mu(q)^{2}}{\phi_{1}(q)}=\left(\prod_{p>2} \frac{(p-1)^{2}}{p(p-2)}\right) \frac{\log y}{4}+O(1)
$$

Hence, by (4), (9), (12) and (13),

$$
\sum_{\substack{x<n<2 x \\ R(n)>0}} 1 \geqslant \frac{\log y}{4 \vartheta(x)} \int_{3}^{2 x} \frac{\min (u, 2 x-u)}{\log u} d u+O\left(x \mathscr{L}^{-1}\right)
$$

The final stage of the proof consists of appealing to a number of density theorems concerning the addition of sets. Whenever a script letter denotes a set, the corresponding italic letter denotes the counting function of the natural numbers contained in the set. Let

$$
\mathscr{A}=\left\{a: 2 a=p_{1}+p_{2}, p_{1} \geqslant 3, p_{2} \geqslant 3\right\} .
$$

Then, by (4) and (14),

$$
\varliminf_{n \rightarrow \infty} \frac{A(n)}{n} \geqslant \frac{1}{2}
$$

Let $\mathscr{B}=\left\{b: b=a_{1}+a_{2}, a_{1} \in \mathscr{A}, a_{2} \in \mathscr{A}\right\}$. Then since $3,4,5 \in \mathscr{A}$, a theorem of Ostmann [31] (Theorem 4.1 of [27]) implies that

$$
\varliminf_{n \rightarrow \infty} \frac{B(n)}{n} \geqslant \frac{3}{5}
$$


If, instead of Ostmann's theorem one uses a deeper theorem of Kneser [21] (see Theorem 18 of [16, Chapter 1] or Theorem 4.2 of [27]) combined with the identity

$$
\sum_{\substack{r=1 \\(r(2 n-r), 2 g)=1}}^{2 g} 1=2 g\left(\prod_{\substack{p|2 g \\ p| 2 n}}\left(1-\frac{1}{p}\right)\right) \prod_{\substack{p \mid 2 g \\ p \nmid 2 n}}\left(1-\frac{2}{p}\right)
$$

and the prime number theorem for arithmetic progressions, then one finds that

$$
\lim _{n \rightarrow \infty} \frac{B(n)}{n}=1 \text {. }
$$

Thus almost every even number is the sum of four primes, which compares with two by the Hardy-Littlewood-Vinogradov method, due to Van der Corput [6], Čudakov [7] and Estermann [13] independently (see also [30], [40]).

By (15), (16) and Dirichlet's box principle, every sufficiently large integer can be written in the form $a+b$ with $a \in \mathscr{A}, b \in \mathscr{B}$. Thus the set $\mathscr{C}=\left\{c: c=\frac{1}{2}(p-1), p \geqslant 3\right\}$ forms an asymptotic basis. Let $\mathscr{D}=\{d: d=a+c, a \in \mathscr{A}, c \in \mathscr{C}\}$. Then, by (15) and a theorem of Rohrbach [34] (an analogue of a theorem of Erdős [12], see Theorem 13 of [16, Chapter 1]),

$$
\varliminf_{n \rightarrow \infty} \frac{D(n)}{n}>\frac{1}{2}
$$

The theorem now follows from (15) and (17) by Dirichlet's box principle.

\section{References}

1. M. B. Barban, “Analogues of the divisor problem of Titchmarsh ”, Vestnik Lenningrad Univ. Ser. Mat. Meh. Astron. 18 (1963), 5-13.

2. _ " On the average order in the generalized prime number theorem ", Dokl. Akad. Nauk UzSSR (1964), 5-7.

3. __ " "The 'large sieve' method and its application to number theory ", Uspehi Mat. Nauk., 21 (1966), 51-102; English translation, Russian Math. Surveys, 21 (1966), 49-103.

4. E. Bombieri, "On the large sieve ", Mathematika, 12 (1965), 201-225.

5. E. F. Cečuro and A. A. Kuzjašev, " The representation of large integers by sums of primes", Issled. Teor. čisel, Saratov, 3 (1969), 46-50.

6. J. G. Van der Corput, "Sur l'hypothèse de Goldbach pour prèsque tous les nombres pairs", Acta Arith., 2 (1937), 266-290.

7. N. G. Cudakov, “ On Goldbach's problem ", Dokl. Akad. Nauk SSSR, 17 (1937), 331-334.

8. —, "On Goldbach-Vinogradov's theorem", Ann. of Math., 48 (1947), 515-545.

9. __ and N. I. Klimov, "Concerning the Šnirel'man constant", Uspehi Mat. Nauk., 22 (1967), 212-213.

10. H. Davenport and H. Halberstam, "Primes in arithmetic progressions ", Michigan Math. J., 13 (1966), 485-489, corrigendum, ibid. 15 (1968), 505.

11. - Multiplicative Number Theory (Markham, Chicago, 1967).

12. P. Erdós, "On the arithmetical density of the sum of two sequences one of which forms a basis for the integers", Acta Arith., 1 (1936), 197-200.

13. T. Estermann, "Proof that almost all even integers are sums of two primes", Proc. London Math. Soc. (1), 44 (1938), 307-314.

14. P. X. Gallagher, “The large sieve ", Mathematika, 14 (1967), 14-20.

15. H. Halberstam and H.-E. Richert, Sieve Methods (Academic Press, London, 1974).

16. - and K. F. Roth, Sequences (Clarendon Press, Oxford, 1966).

17. H. Heilbronn, E. G. H. Landau and P. Scherk, "Alle grossen Zahlen lassen sich als Summe von höchstens 71 Primzahlen darstellen ", Čas. mat. fy's., 65 (1936), 117-140. 
18. C. Hooley, "On the Barban-Davenport-Halberstam theorem. I", J. Reine Angew. Math., $274 / 5$ (1975), 206-223.

19. N. I. Klimov, “Apropos the computations of Šnirel'man's constant ", Volž. Mat. Sb., 7 (1969), $32-40$.

20. N. I. Klimov and L. F. Kondakova, “Certain additive problems", Volž. Mat. Sb., 7 (1969), 41-44 (loose errata).

20a. — , G. Z. Pil'tjaĭ and T. A. Septickaja, “ Eine Abschätzung der absoluten Konstante im Goldbach-Snirel'manschen Problem ", Issled. Teor. Čisel., Saratov 4 (1972), 35-51.

21. M. Kneser, "Abschätzung der asymptotischen Dichte von Summenmengen ", Math. $Z$., 58 (1953), 459-484.

22. E. G. H. Landau, "Die Goldbachsche Vermutung und der Schnirelmannsche Satz", Nachr. Ges. Wiss. Göttingen, (1930), 255-276.

23. — Uber einige neuere Fortschritte der additiven Zahlentheorie (Cambridge Tract No. 35, London, 1937).

24. A. F. Lavrik, " On the representation of numbers as the sum of primes by Šnirel'man's method ", Izv. Akad. Nauk UzSSR Ser. Fiz.-Mat. Nauk (1965), No. 3, 5-10.

25. Ju. V. Linnik, "On the possibility of a unique method in certain problems of 'additive' and 'multiplicative' prime number theory", Dokl. Akad. Nauk SSSR (N. S.), 49 (1945), 3-7.

26. —_, "A new proof of the Goldbach-Vinogradov theorem ", Mat. Sb. (N.S.), 19 (1946), 3-7.

27. H. B. Mann, Addition Theorems (Interscience, Wiley, New York, 1965).

28. H. L. Montgomery, " Primes in arithmetic progressions", Michigan Math. J., 17 (1970), 33-39.

29. —-, Topics in Multiplicative Number Theory (Springer Lecture Notes, 227, Berlin, 1971).

30. — and R. C. Vaughan, "The exceptional set in Goldbach's problem ", Acta Arith., 27 (1975), 353-370.

31. H.-H. Ostmann, "Verfeinerte Lösung der asymptotischen Dichtenaufgabe", J. Reine Angew. Math., 187 (1950), 183-188.

32. G. Ricci, "Su la congettura di Goldbach e la constante di Schnirelmann", Boll. Un. Ital., 15 (1936), 183-187.

33. _ـ " "Su la congettura di Goldbach e la constante di Schnirelmman, I mem.,", Ann Scuola Norm. Sup. Pisa (2), 6 (1937), 71-90, II mem., ibid. 91-116.

34. H. Rohrbach, " Einige neuere Untersuchungen über die Dichte in der additiven Zahlentheorie ", Jber. Deutsch Math.-Verein., 48 (1938), 199-236.

35. N. P. Romanov, "The Goldbach problem," Izv. NII math. mech. Un-ta, Tomsk, 1 (1935), 34-38.

36. H. N. Shapiro and J. Warga, "On the representation of large integers as sums of primes", Comm. Pure Appl. Math., 3 (1950), 153-176.

37. H. Siebert, Datstellung als Summe von Primzhalen (Diplomarbeit, Marburg, 1968).

38. L. G. Snirel'man, “On additive properties of numbers ", Izv. Donskowo Politeh. Inst., 14 (1930), 3-28.

39. —_ "Uber additiven Eigenschaften von Zahlen ", Math. Ann., 107 (1933), 649-690.

40. R. C. Vaughan, " On Goldbach's problem ", Acta Arith., 22 (1972), 21-48.

41. A. I. Vinogradov, "On the density hypothesis for Dirichlet L-functions", Izv. Akad. Nauk SSSR Ser. Mat., 29 (1965), 903-934, correction, ibid. 30 (1966), 719-720.

42. I. M. Vinogradov, "Representation of an odd number as a sum of three primes", Dokl. Akad. Nauk. SSSR, 15 (1937), 169-172.

43. _ "Some theorems concerning the theory of primes", Recueil Math. 2 (44), 2 (1937), 179-195.

44. - The method of trigonometrical sums in the theory of numbers, translated from the Russian, revised and annotated by K. F. Roth and A. Davenport (Interscience Publishers, 1954).

45. Yin, Wen-lin, "Remarks on the representation of large integers as sums of primes", Acta Sci. Natur. Univ. Pekinensis, 3 (1956), 323-326; English translation, Note of the representation of large integers as sums of primes, Bull. Acad. Polon. Sci. Cl. III, 4 (1956), 793-795.

Imperial College, London.

University of Michigan, Ann Arbor. 